\title{
Experimental Studing of Mechanical-and- Physical Properties of Rubber during Ageing
}

\author{
Svetlana Polukoshko \\ Ventspils University College \\ Engineering Research Institut "VSRC" \\ Ventspils, Latvia \\ pol.svet@inbox.lv
}

\author{
Andris Martinovs \\ Rezekne Academy of Technologies \\ Rezekne, Latvia \\ andris.martinovs@rta.lv
}

\author{
Edgars Zaicevs \\ Rezekne Academy of Technologies \\ Rezekne, Latvia \\ edgars.zaicevs@rta.lv
}

\begin{abstract}
Elastomeric materials, both natural rubber and synthetic, are widely used in industry and civil engineering, due to their unique properties: high elasticity, low volume compressibility, capability to absorb and dissipate input energy, a linear relationship between stress and strain up to strain of $15 \% \div 20 \%$, resistance to aggressive environmental factors. Different kind of compensation devices, vibration dampers, shock absorbers are fabricated from rubber materials.

At the same time the elastomeric materials nonreversible change their properties over time, this disadvantage is called ageing.

In given paper the results of experimental studying of the influence of aging on the physical-and-mechanical properties of polyurethane rubber is presented. The samples of cylindrical form were prepared from soft flexible polyurethane rubber Xenias PX30 and subjected to the artificial ageing. Accelerated aging of samples was fulfilled in accordance with European standard ISO 188:2011 (Rubber, vulcanized or thermoplastic - Accelerated ageing and heat resistance tests).

The changing of volume, Shore A hardness, elastic rebound coefficient and static elasticity modulus under compression were investigated. Experiments showed the volume decrease, hardness shore increasing, elastic rebound increase and compression modulus under static loading increasing. This data are necessary for correct designing of the compensation devices to provide their working properties during all service life.
\end{abstract}

Keywords-accelerated ageing, elastomers, hardness, compression static loading, stiffness.

\section{INTRODUCTION}

Natural and synthetic rubber is very important structural materials, which is widely used in various sectors of the economy and in everyday life: vehicle manufacturing, shipbuilding, cable and electrical industry, building industry, products for contact with food and drinking water, medical, pharmaceutical, hygiene products and products for children.

Such extensive use the elastomeric materials received primarily due to their unique properties: ability to deform significantly at relatively low stresses, restore the original shape after removing the load, absorb and dissipate mechanical energy during deformation. They have high strength, low volume compressibility (change shape

under mechanical loading, keeping almost a constant volume); low residual deformation; high wear resistance, resistance to ultraviolet radiation and radiation [1].

Thereby rubbers are widely used in engineering and industry where products operate in "harsh" conditions for such technical products as seals of any type, bushings, plugs, sockets, valves and plugs, shock absorbers, rollers.

The main disadvantage of rubber materials is their ability to change their properties significantly under the influence of external factors of a mechanical and nonmechanical nature. These changing are associated with corresponding changes in the structure and may be reversible and irreversible.

Irreversible changes may be the result of a number of mechanochemical and chemical processes activated by mechanical stress. Irreversible destruction of the structure under the action of mechanical forces is the greater, the lower the rate of relaxation processes. With repeated deformations, this leads to corresponding changes in the structure and properties of rubber.

One of non-mechanical factors affecting the properties of rubber is the effect of heat. It causes reversible changes in the structure and properties associated with an increase in the energy of thermal motion. Along with this, prolonged exposure of rube to heat, especially in a chemically active medium, may lead to irreversible changes, called ageing.

Ageing is the irreversible change in the properties of a rubber under the action of heat, light, oxygen, air, ozone, etc, or corrosive media, i.e. mostly non-mechanical factors. Ageing is activated if the rubber is simultaneously exposed to mechanical loads [2] - [4].

Ageing tests are carried out by withstanding rubber under various conditions. Experiments on accelerated ageing test are regulated by ISO standards [5] - [7]. With atmospheric aging in the open air or thermal ageing in an environment of hot air, the result of the test is assessed by the coefficient of aging, which is the ratio of changes in the performance of some properties, most often tensile strength and relative elongation at break, to the corresponding indicators before aging [8]. The smaller the change in properties during ageing and the coefficient 
of aging, the higher the resistance of rubber to ageing.

As soon scope of our study is application of elastomeric materials for technical products like mechanical seals, joints, etc., in machinery we estimate the change of compression elastic modulus, hardness Shore A, elastic rebound coefficient and volume change during accelerated ageing of polyurethane rubber. The Xencas PX series is a range of industry leading flexible polyurethane rubbers which cure to hardness from 30 to 90 Shore A whilst retaining excellent tear strength and resilience. Xencast PX resins are ideal for product applications requiring a flexible yet durable material including soft-grip handles, protective cases, rests, training weapons, tools, wheels, bushes and much more. PX series resins can also be used to produce durable, flexible molds for concrete casting. Utilizing the latest polymer technology, Xencast PX30 is able to create flexible PU rubber parts with superior tear strength, abrasion and wear resistance and chemical tolerance.

Suggested uses of Xencast PX rubber are soft gaskets and seals, therefore the main goal of this work is the investigation of this product under pure compression and the changing of its property in time.

\section{Materials AND Methods}

\section{A. Material and specimens}

Material, used in research, is Easy Composites Ltd Xencast PX30 soft flexible polyurethane rubber. Cured properties of this product: Shore A hardness $30-35$, tensile strength $0.7-1.2 \mathrm{MPa}$, elongation at break $100-155 \%$, maximum operating temperature $80^{\circ} \mathrm{C}$. These properties are given by manufacturer, elastic modules at static load $E=3.20 \pm 0.10 \mathrm{MPa}$ was obtained earlier by compression test with machine Zwick / Roell Z-150 in RTA laboratory.

Test specimens were prepared of cylindrical shape with initial dimensions of a diameter $39 \mathrm{~mm}$ and a height $13 \mathrm{~mm}$. Samples were made in accordance to standard ISO 23529, 2010 [9].

\section{B. Testing methodology and machinery}

All equipment for rubber testing were calibrated accorging to the guide ISO 18899:2004 [10].

The artificial ageing of polyurethane rubber samples was performed according to DIN 53508: 2000 in the thermostat Memmert UNE 400 [11].

The oven Memmert UNE 400 (maximal temperature $+250^{\circ} \mathrm{C}$ ) was produced in Germany with the use of high-grade materials and the application of the latest manufacturing techniques. Samples were subjected to heating under $100^{\circ} \mathrm{C}$ during $14,24,56,72$, 120 and 168 hours. The specimens sizes after heating decrees.

The relative change of volume during heating time is calculated as:

$$
\theta=\frac{V-V_{0}}{V_{0}} \cdot 100 \%
$$

where: $V_{0}$ - the volume of the sample before ageing, $V$ is the volume of the sample after aging.
Shore A hardness measurement was performed in accordance with DIN 53505: 2000 Testing of rubber - Shore A and Shore D hardness test [12] and ISO 484:2018 [13].

The rebound elasticity was determined according to DIN 53512: 2000, using Schob pendulum in case of direct central impact shown in Fig. 1 [14].

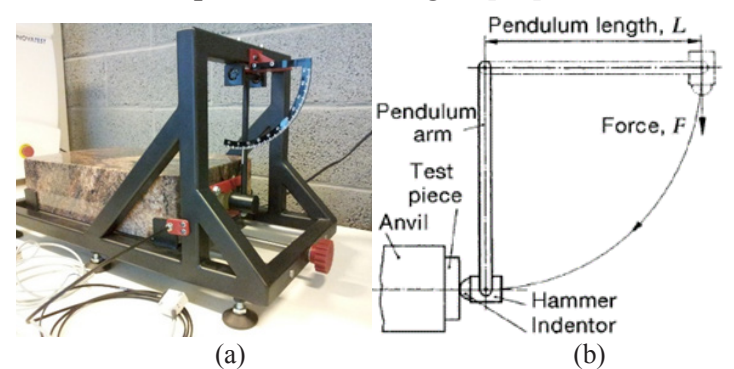

Fig. 1. Rebount resilience test: .(a) testing rig, (b) principle of test

Rebound resilience $R$ is the ratio of energy returned $E$ to energy applied $E_{0}$ Rebound resilience:

$R=\frac{E}{E_{0}}=\frac{m g h}{m g h_{0}}=\frac{h}{h_{0}}=\frac{L \cdot(1-\cos \alpha)}{L \cdot\left(1-\cos \alpha_{0}\right)}=\frac{1-\cos \alpha}{1-\cos \alpha_{0}}$,

where: $m$ - indentoe mass,

$$
\begin{aligned}
& g \text { - gravity acceleration, } \\
& \alpha \text {-coner of pendulum deviationion. }
\end{aligned}
$$

If $\alpha 0=900 \mathrm{R}=(1-\cos \alpha) \cdot 100 \%$ in perctntage.

The elasticity modulus is determined using the pure compression test data. The compression test was performed with Zwick / Roell Z-150 universal tensioning machine (Fig. 2 a); strain rate $10 \mathrm{~mm} / \mathrm{min}$; maximum deformation $18 \%$.

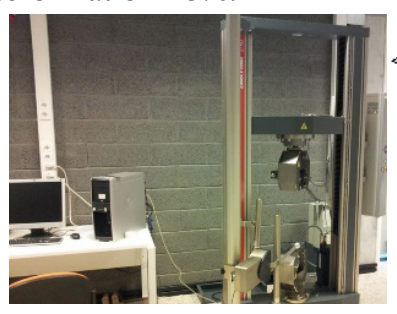

(a)

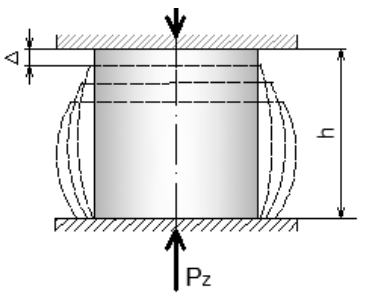

(b)
Fig. 2. Compressing testing: (a) Zwick / Roell Z-150 universal testing machine, (b) scheme of sample deformation.

The mechanical stress is to be. calculated in relation to the true cross-section. The deformative behavour of spesimens under compression depend on boundary conditions, i.e. contact friction between steel and rubber (usually friction factor $f=0.2-0.3$ ) and deformed spesimen will be of barrel-shaped cylinder (Fig. 2b). Straight circular cylider may save its form after compression between two rigid plates only if $f=0$.

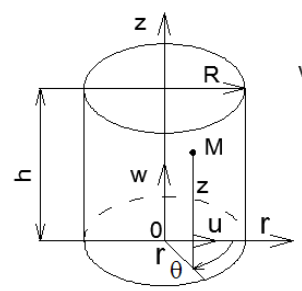

(a)

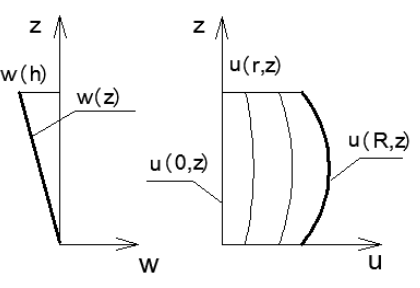

(b)
Fig. 3. Cylindrical coordinate system (a); displacement functions $w(z)$ and $u(r, z)(\mathrm{b})$ 
The deformed shape of the cylinder may be defined using displacement fu nctions, which are required for minimization of the total potential energy functional in Ritz method [15]. For axially symmetric problems cylindrical coordinates system are chosen and the sought -for functions of displacement $(u, v, w)$ are set as the functions of the coordinates $(r, \theta, z)$. Coordinate system and scheme of rubber block displacement are shown in Fig. 3.

Displacement functions must satisfy the main boundary conditions, as well as the symmetry and the presence of extremum must be taken into account; in our case for simplicity we choose:

$$
u=C_{1} f_{1}(z), v=0, w=C_{2} f_{2}(z) \text { and } f_{1}=\frac{\partial f_{2}}{\partial z}
$$

Contact geometrical boundary conditions are the next:

$$
\begin{gathered}
u(r, z=0) \neq 0, u(r, z=h) \neq 0, w(r, z=0)=0, \\
w(r, z=h)=-\Delta .
\end{gathered}
$$

The displacement functions $u, v, w$ along the axis of cylindrical coordinate system $r, \theta, z$ are chosen as:

$$
\begin{aligned}
u(r, z) & =C_{1} r\left(z^{2}-z h-h^{2}\right), \quad v(r, z)=0, \\
w(r, z) & =C_{2}\left(\frac{z^{3}}{3}-\frac{z^{2} h}{2}-z h^{2}\right)
\end{aligned}
$$

Relative deformation functions along cylindrical coordinate axis $r, \theta, z$ are:

$$
\varepsilon_{r}=\frac{\partial u}{\partial r}, \varepsilon_{\theta}=\frac{u}{r}, \varepsilon_{z}=\frac{\partial w}{\partial z} ;
$$

For incompressible material (Poisson's ratio $\mu=0.5$ ):

$$
\varepsilon_{r}+\varepsilon_{\theta}+\varepsilon_{z}=0
$$

Constants $C_{1}$ and $C_{2}$ as functions of $\Delta$ are determined from the boundary conditions (4) and conditions (7):

$$
\begin{gathered}
C_{1}=-0.429 \frac{\Delta}{h^{3}}, C_{2}=0.858 \frac{\Delta}{h^{3}} . \\
D_{Z}=_{0, h}=D\left(1+0.429 \frac{\Delta}{h}\right), \\
D_{Z=\frac{h}{Z}}=D\left(1+0.536 \frac{\Delta}{h}\right) .
\end{gathered}
$$

True cross-section areas for each step of loading are determined with (9).

\section{Results AND Discussion}

The main results of the investigation are given below. In Fig. 4 - 7 the plots of dependence of physicalmechanical properties of elastomer samples on the time of heating under temperature $T=100^{\circ}$ are presented. In Fig. 8 - 12 show compressive stress - strain diagrams for samples without aging and after 1, 3, 5 and 7 days heating.

During heating of the cylindrical samples they decrease in sizes, both height and diameter, shrink and their volume reduce up to $35 \%$ (Fig. 4).

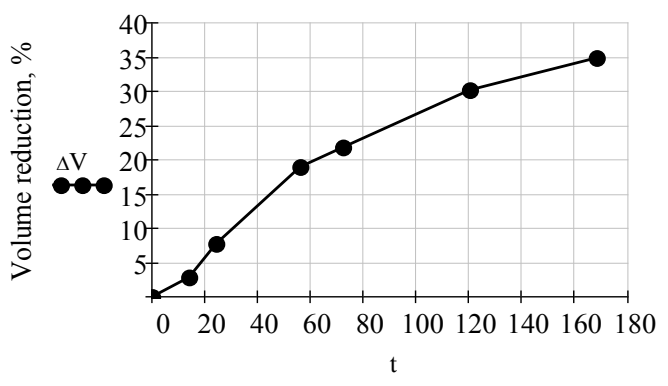

Time of heating, hours

Fig. 4. Volume changing depemdence on time of heating under temperature $T=100^{\circ}$.

The elasticity of polymers diminish in time, so Shore A hardness of the testing samples increase from 40 to 52.7 (Fig. 5). In accordance to scale type A this material is soft and middle soft.

Rebound resilience value decrease during time heating from 79 to $68 \%$ (Fig. 6).

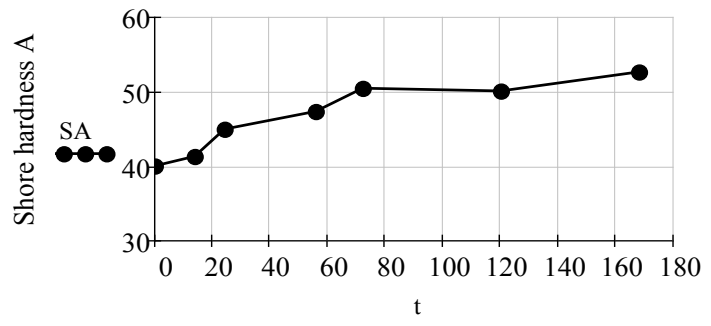

Time of heating, hours

Fig. 5. Shore hsrdness A dependce on the time $t$ of heating under temperature $T=100^{\circ}$.

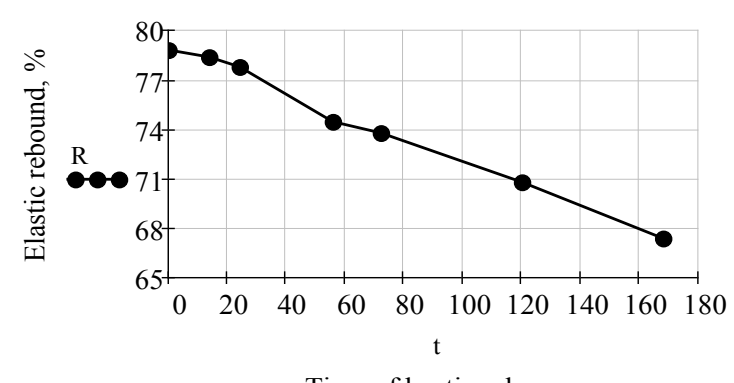

Time of heating, hours

Fig. 6. Rebound resiliens value dependce on the time $t$ of heating under temperature $T=100^{\circ} \mathrm{C}$.

In Fig. 7 experimental and approximated curve of the modulus of elastisity $E$ and Eap are shown as function of time heating. Experimental value of elastisity modulus is calculated taking into account the changing of the cross section area during compression. i.e. $E$ is so-called apparent modulus. Approximated curve of modulus of elasticity is represented as a power function (10).

$$
E_{\text {ap }}(t)=0.048 t^{0.71}+3.458
$$




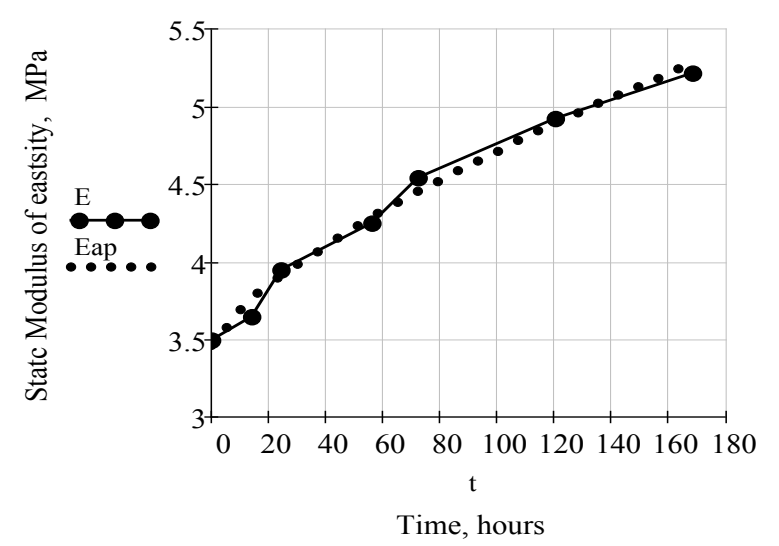

Fig. 7. Static compressive modulus of elasticity dependence on time of heating under $T=100^{\circ} \mathrm{C}$.

The constants in equation are defined using Mathcad-15 program with help of built-in function for curves adjusting and fitting.

This equation allows easy to estimate elastic modulus value and, consequently, deformation for any time interval.

In Fig. 8 - 12 the plots of compression stress - strain dependence for samples without ageing, samples with 1 , 3,5 and 7 days of heating are presented.

In these plots stress $\sigma_{0}$ with initial cross-section area and stress $\sigma$, taking into account cross-section area change, are given. Stress $\sigma_{i}$ is approximated by straight lines $f_{i}\left(\varepsilon_{i}\right)$ in accordance with (11) - (15), given below each plot. These straight lines in the plot show the linear relationship between stress and strain and constant modulus of elasticity. The slope of these straight lines indicate the value of modulus of elasticity; it is seen from the equations that elasticity modulus increase with ageing and remain constant during experiments up to strain $16-18 \%$.

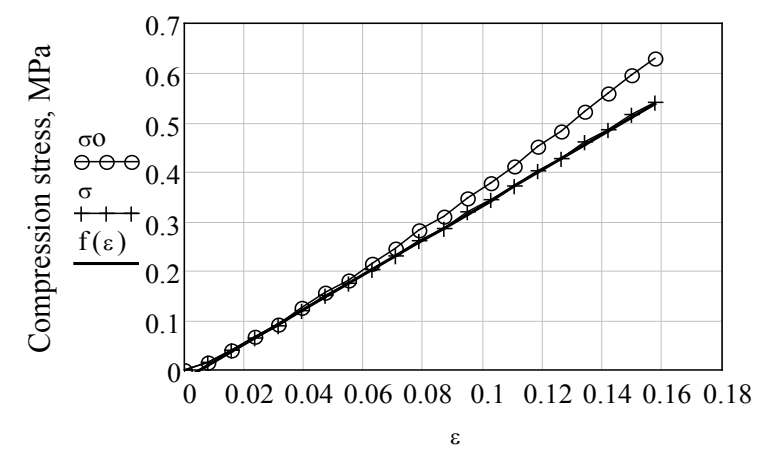

Relative strain

Fig. 8. Static compression stress dependence on relative strain for sample without heating.

$$
f(\varepsilon)=3.51 \varepsilon 1-0.02
$$

For the samples without ageing dependence " $\sigma-\varepsilon$ " is linear during all period of loading (Fig. 8). This is true for all elastomeric materials up to strain of $15 \% \div 20 \%$.

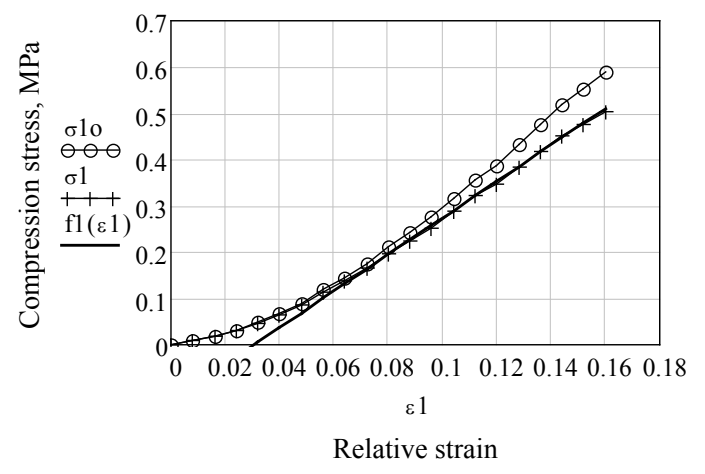

Fig. 9. Static compression stress dependence on relative strain for sample after 1 day heating under $T=100^{\circ} \mathrm{C}$.

$$
\mathrm{f} 1(\varepsilon 1)=3.95 \varepsilon 1-0.12
$$

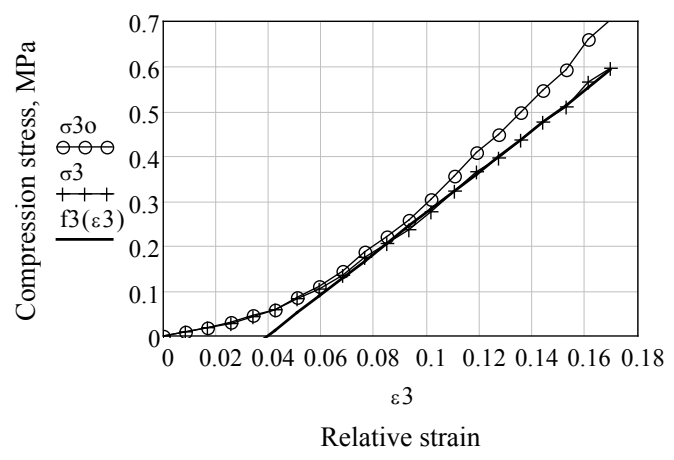

Fig. 10. Static compression stress dependence on relative strain for sample after 3 days heating under $\mathrm{T}=100^{\circ} \mathrm{C}$.

$$
\mathrm{f} 3(\varepsilon 3)=4.54 \varepsilon 6-0.18
$$

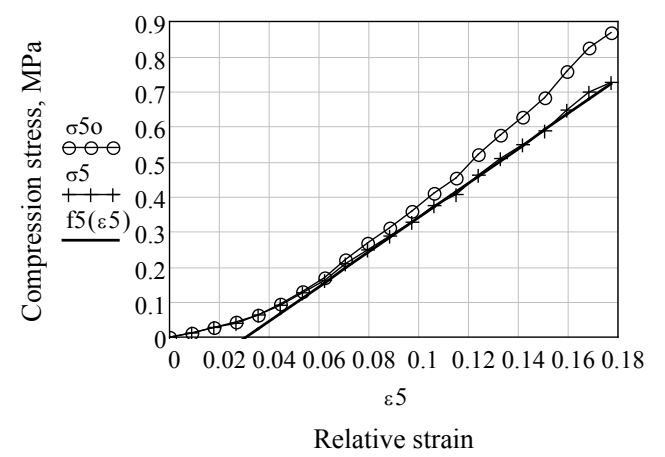

Fig. 11. Static compression stress dependence on relative strain for sample after 5 days heating under $T=100^{\circ} \mathrm{C}$.

$$
\mathrm{f} 5(\varepsilon 5)=4.92 \varepsilon 5-0.15
$$

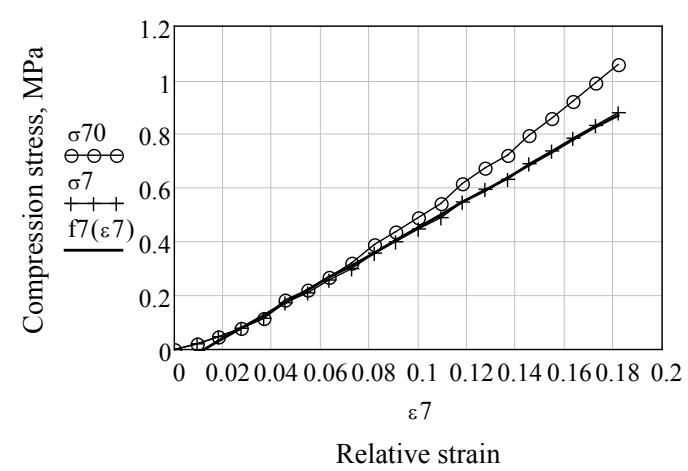

Fig. 12. Static compression stress dependence on relative strain for sample after 7 days heating under $T=100^{\circ} \mathrm{C}$. 


$$
\mathrm{f} 7(\varepsilon 7)=5.12 \varepsilon 7-0.07
$$

After 7 days heating the elastic compression modulus reach 5.12 $\mathrm{MPa}$, which is almost $50 \%$ increasing from the initial value $3.51 \mathrm{MPa}$. Cross-sectional area decrease from $1207 \mathrm{~mm}^{2}$ till $908 \mathrm{~mm}^{2}$ in undeformed state and from $1408 \mathrm{~mm}^{2}$ till $1089 \mathrm{~mm}^{2}$ after deformation of $2 \mathrm{~mm}$.

\section{CONCLUSIONS}

In this work the experimental studding of the influence of aging on the physical and mechanical properties of rubber is performed. The samples of cylindrical form were prepared from soft flexible polyurethane rubber Xencast PX30 and subjected to the artificial aging. Accelerated aging of samples was fulfilled in accordance with European standard ISO 188:2011 Rubber, vulcanized or thermoplastic - Accelerated ageing and heat resistance tests [5].

The changing of volume, hardness Shore A, elastic rebound coefficient and static elasticity modulus under compression were investigated. Experiments showed the volume decrease, hardness shore increasing, elastic rebound increase and compression static modulus increasing.

Influence of ageing on dynamic characteristics of polyurethane rubber Xenias PX30 will be developed in the next step of this study, based on works [16] - [18]

\section{REFERENCES}

[1] A. N. Gent, Engineering with Rubber: How to Design Rubber Components. Munich, Carl Hanser Verlag, 2011.

[2] Brown R. P., Bulter T. and Hawely S.W. Ageing of Rubber: Acceleration Heat Ageing Test Results, Smither Rapra Publishing, 2001. Available: https://epdf.tips/ageing-of-rubber-acceleratedheat-ageing-test-results.html

[3] Reincke et. all "Experimental characterization of the ageingr esistanse of elastomers", in: Constitutive Models for Rubber IX, Marvalova \& Petrikova, Ed. Taylor \& Francis Group, London, UK, 2015, pp. 33-37.

[4] A, Martinovs, "Mechanical properties of elastomers forecasting and principles of accelerated experiment," D.S. thesis, Riga Technical University, Riga, EU, Latvija, 2005.

[5] ISO 188:2011* Rubber, vulcanized or thermoplastic - Accelerated ageing and heat resistance tests.

[6] ISO 11346 Rubber, vulcanized or thermoplastic - Estimation of life-time and maximum temperature of use.

[7] ISO/TR 9272 Rubber and rubber products - Determination of precision for test method standards

[8] ISO 37:2017(E) Rubber, vulcanized or thermoplastic Determination of tensile stress-strain properties.

[9] ISO 23529: 2010 Rubber - General procedures for preparing and conditioning test pieces for physical test methods.

[10] ISO 18899:2013, Rubber - Guide to the calibration of test equipment.

[11] DIN 53508: 2000. Prüfung von Kautschuk und Elastomeren. Künstliche Alterung. Testing of rubber and elastomers. Artificial ageing of rubber. ISO 18899:2004,

[12] DIN 53505:2000. Prüfung von Kautschuk und Elastomeren. Härteprüfung nach Shore A und Shore D. Testing of rubber Shore A and Shore D hardness test.

[13] ISO 48-4:2018 Rubber, vulcanized or thermoplastic -Determination of hardness -- Part 4: Indentation hardness by durometer method (Shore hardness).

[14] DIN 53512: 2000 Determining the rebound resilience of rubber using the Schob pendulum.

[15] S. Polukoshko, V. Gonca, A. Martinovs, S. Sokolova, "Boundary conditions influence on compressive stiffness of elastomeric isolators", Proceedings of the 15-th international scientific conference Engineering for Rural Development 2016, Volume 15 May 25-27, 2016, Jelgava, p. 924-931. Available: http://www. tf.1lu.lv/conference/proceedings2016/Papers/N137.pdf

[16] S. Polukoshko, A. Martinovs, S. Sokolova. "Aging, Fatigue and Durability of Rubber Vibration Isolation Elements." Proceeding of the 11th International Scientific and Practical Conference "Environment. Technology. Resources.", Volume III, pp. 269-275.

[17] S. Polukoshko, A. Martinovs, E. Zaicevs, "Influence of rubber ageing on damping capacity of rubber vibration absorber", in: Vibroengineering PROCEDIA, September 2019, Volume 19, pp. 103-109.

[18] Martinovs A., Polukoshko S., Apeinans E., Zaicevs E. "Determination constants of 4-element rheological model with rebound resilience method." Proceedings of the 16-th international scientific conference Engineering for Rural Development 2017, Volume 16 May 24-26, 2017, Jelgava, p. 924-931.Available: http://tf.llu.lv/conference/proceedings2017/Papers/N189.pdf 